\title{
ESTUDIO DE LAS POBLACIONES DE CANGREJO UTILIZADAS COMO CARNADA EN LAS ISLAS CANARIAS: SITUACIÓN ACTUAL, INFLUENCIA DEL MARISQUEO Y TIPO DE HÁBITAT
}

\author{
Ana Forner*, María Bas-Silvestre, Adrián Martín-Hernández, \\ Daniel Álvarez-Canali \& Neliann Collazo
}

\section{RESUMEN}

Se estudió la diferencia de abundancia, talla y biomasa de las especies de cangrejo usadas como carnada entre las localidades de Punta del Hidalgo y Finca El Apio, entre las que se asume diferente presión marisquera. Para ello se registró la talla, sexo y peso de los taxones de interés y se trató de establecer una relación entre la abundancia y el tipo de hábitat. En ambas localidades se recolectó mayor número de machos de los géneros Pachygrapsus y Xantho. Percnon gibbesi y Pachygrapsus spp. alcanzan tallas medias mayores en la Punta del Hidalgo, mientras que Xantho spp. cuenta con tallas similares en las dos zonas. El número de hembras ovígeras se mostró bajo pese a ser época de veda. Se determina que no existen diferencias significativas entre las dos localidades en cuanto al marisqueo, pero sí diferencias de abundancia a nivel de hábitat. A nivel individual, el género Pachygrapsus contó con mayor presencia en áreas donde predominan rocas de tamaño grande. Se recomienda un seguimiento de las poblaciones en periodos dentro y fuera de veda, así como desarrollar estudios centrados en la biología y ecología de dichas especies.

Palabras clave: carnada, hábitat, intermareal, marisqueo, Pachygrapsus, Xantho, Percnon.

\section{STUDY OF THE CRAB POPULATIONS USED AS BAIT IN THE CANARY ISLANDS: ACTUAL STATUS, HARVESTING AND HABITAT INFLUENCE}

\begin{abstract}
Differences in abundance, size and biomass were studied in crab species used as bait for fishing in two locations: Punta del Hidalgo and Finca El Apio, assuming different shellfishing pressure between them. Thus, size, sex and weight of the animals were registered in order to establish a relationship between the abundance and type of habitat. A higher number of Pachygrapsus spp. and Xantho spp. males was collected in both locations. P gibbesi and Pachygrapsus spp. reached higher shell-length values in Punta del Hidalgo, but Xantho spp. had similar length in both areas. The number of ovigerous females was low despite being harvesting ban period. No significant differences in terms of harvesting were found, but it differs according to habitat type. Individually, the genus Pachygrapsus had greater presence in areas dominated by large rocks. It is recommended to continue with the population monitoring of crabs used as bait in and out harvesting ban period and develop studies focused on the biology and ecology of these species.
\end{abstract}

Keywords: bait, habitat, intertidal, Pachygrapsus, shellfishing, Xantho, Percnon. 


\section{INTRODUCCIÓN}

Uno de los principales efectos asociados a la antropización en las zonas costeras es la explotación de los recursos marinos, y más concretamente el marisqueo intermareal (Freire y García-Allut 2000; Ferns et al. 2000; Noguera y Riera 2011). La recolección selectiva de individuos en función de su tamaño puede influir sobre la demografía, abundancia y biomasa de la población, comportamiento, ciclo vital y genética de las especies recolectadas (Fenberg y Roy 2008).

En el archipiélago canario, las especies de crustáceos decápodos Percnon gibbesi (H. Milne Edwards, 1853) y las relativas al género Xantho (Leach, 1814) y Pachygrapsus (Randall, 1840) son utilizadas como carnada para la pesca profesional y recreativa (BOC n. ${ }^{\circ}$ 093, 2 de mayo 2011). El marisqueo incontrolado, junto con la contaminación y degradación del litoral, convierte a estos decápodos en vulnerables (Bonnet y Rodríguez 1992; González 2016).

Estas especies de interés marisquero en el litoral canario habitan en sustratos rocosos y rocoso-arenosos, bajo piedras y callaos y en intersticios de rocas sumergidas, en ambientes protegidos, plataformas, charcos intermareales y plataformas dominadas por especies de macroalgas como Corallina y Cystoseira (González 1995). Spivak et al. (2010) demostraron que los cangrejos del género Xantho muestran preferencia por piedras grandes dispuestas sobre fondo rocoso consistente en pequeños callaos, siendo menos frecuentes en zonas dominadas por arena. Por otra parte, la especie Percnon gibbesi se encuentra frecuentemente en charcos intermareales bajo piedras de gran tamaño o en grietas y en el infralitoral somero (Deudero et al. 2005). En cuanto a su comportamiento durante la marea baja, el género Xantho se muestra inactivo y permanece bajo rocas, mientras que las especies de Pachygrapsus se comportan de forma activa (Spivak et al. 2010). Se ha observado que la especie $P$. gibbesi es activa durante el día, contando con mayor actividad de alimentación durante el atardecer (Deudero et al. 2005).

El ciclo reproductivo de los géneros Xantho y Pachygrapsus es estacional (Flores y Paula 2002a; Flores y Paula 2002b; Spivak et al. 2010), registrándose picos sucesivos de abundancia de hembras ovígeras durante la primavera y el verano (de abril a agosto), aunque las hembras probablemente producen múltiples puestas durante el periodo entre mudas. Para Percnon gibbesi la mayor abundancia de hembras ovígeras se muestra también a principios de verano (Paula 1989; Sciberras y Schembri 2007; Noguera y Riera 2011).

Los pocos estudios realizados hasta la fecha sobre el estado de las poblaciones de estos decápodos en el litoral de Canarias hacen que trabajos como éste sean de especial interés para la gestión y conservación de los recursos litorales. Noguera y Riera (2011) observaron síntomas de sobreexplotación asociada al marisqueo en

* Máster de Biología Marina: Biodiversidad y Conservación. Universidad de La Laguna, San Cristóbal de La Laguna, Tenerife, islas Canarias, España. Autor para la correspondencia: alu0101004938@ull.edu.es. 


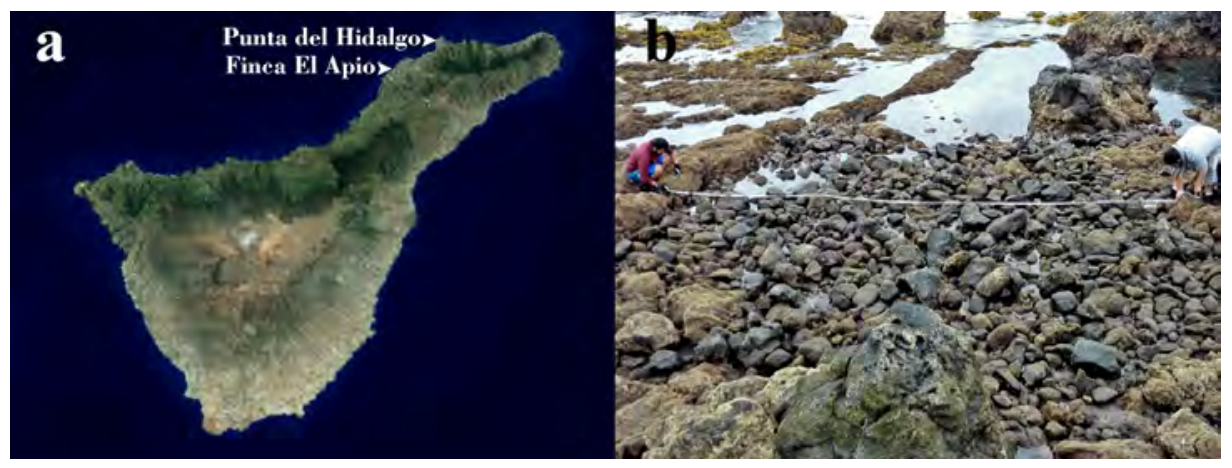

Figura 1. a. Localización de las áreas de estudio: Finca El Apio $\left(28,529187^{\circ} \mathrm{N} 16,40683^{\circ} \mathrm{O}\right)$ y Punta del Hidalgo $\left(28,576691^{\circ} \mathrm{N} 16,329255^{\circ} \mathrm{O}\right) \mathrm{i}$ en Tenerife (Islas Canarias) (imagen tomada de GRAFCAN). b. Una de las áreas de muestreo seleccionadas.

las poblaciones del género Xantho en la isla de Lanzarote, notificando la pequeña talla de los individuos y la escasez de individuos adultos y hembras ovígeras. El Gobierno de Canarias, mediante el Boletin Oficial de Canarias del 2 de mayo de 2011, ha establecido volúmenes máximos de captura, zonas del litoral acotadas para la recuperación de poblaciones y periodos de veda. Para las especies Percnon gibbesi y las relativas a los géneros Xantho y Pachygrapsus el marisqueo queda prohibido anualmente entre el 1 de diciembre y el 31 de marzo.

En este estudio se determinó si las poblaciones de cangrejo utilizadas como carnada se ven afectadas por el marisqueo en el norte de la isla de Tenerife. Para ello se estudió y comparó la talla y abundancia de estas especies entre la Punta del Hidalgo, zona muy accesible y frecuentada por pescadores, en la que se asume alta presión de marisqueo, y la Finca El Apio, área en la que se espera menor presión de recolección debido a la dificultad de acceso. Además, se examinó la relación entre la abundancia de estas especies y el tipo de hábitat que ocuparon.

\section{MATERIAL Y MÉTODOS}

Fueron seleccionadas como área de estudio las localidades de la Punta del Hidalgo y la Finca El Apio, en el municipio de San Cristóbal de La Laguna (Tenerife, islas Canarias) (figura 1a). La Punta del Hidalgo y la Finca El Apio constituyen dos rasas rocosas localizadas en el norte de Tenerife de gran interés para el estudio de los ecosistemas litorales, debido a sus dimensiones y al gran número de especies que podemos encontrar en ellas. Estas plataformas difieren en gran medida en la facilidad de acceso que presenta cada una de ellas, lo que supone una aparente diferencia en cuanto a la facilidad de explotación de sus recursos (Alfonso et al. 2015). Se seleccionaron tres áreas dentro de ambas localidades que reunieran las condiciones ambientales propicias para el estudio (figura $1 \mathrm{~b}$ ), haciendo tres réplicas en el caso de la Punta del 


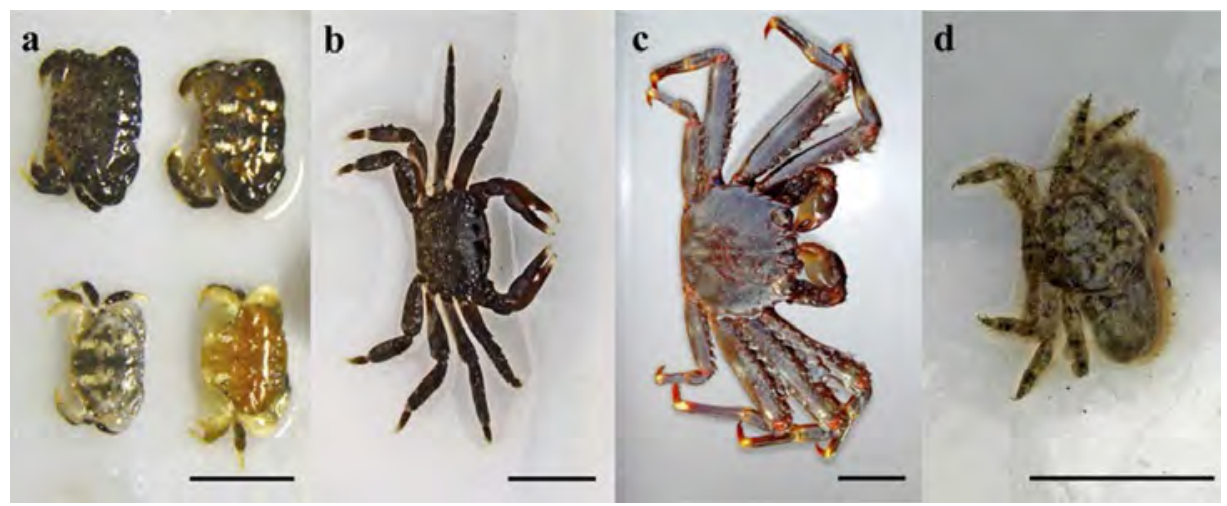

Figura 2. Especies objeto de estudio, de izquierda a derecha: a. Xantho spp.,

b. Pachygrapsus spp. c. Percnon gibbesi y d. Porcellana platycheles (escala $=10 \mathrm{~mm}$ ).

Hidalgo y dos en la Finca El Apio debido a las limitaciones impuestas por el espacio y la marea. Para cada una de estas réplicas se midió el área de la zona de muestreo.

Durante el mes de febrero de 2016 se procedió a muestrear cada área de estudio y sus correspondientes réplicas. Durante 30 minutos, cuatro investigadores se dedicaron a recolectar individuos de las especies Percnon gibbesi y relativas a los géneros Xantho y Pachygrapsus entre las rocas. Por su abundancia, individuos de Porcellana platycheles también se recolectaron, aunque no es considerada una especie de interés marisquero (figura 2). Todos los ejemplares fueron sexados, indicando si las hembras eran ovígeras, y se midió el ancho máximo del cefalotórax con un calibrador. Algunos individuos de Xantho y Pachygrapsus fueron llevados al laboratorio para registrar su peso en la báscula de precisión y posteriormente fueron devueltos al mar. Se establecieron cuatro clases de individuos en función del sexo: machos, hembras no ovígeras, hembras ovígeras e indeterminados (para aquellos animales en los que fue imposible determinar el sexo).

Con el objetivo de describir el hábitat de las especies de interés se seleccionaron al azar 15 rocas de cada zona de muestreo y se midió su perímetro, lo que permitió definir dos tipos de hábitat, uno dominado por rocas pequeñas (con menos de seis rocas con perímetro superior a $50 \mathrm{~cm}$ ) y otro por rocas grandes (con seis o más rocas de más de $50 \mathrm{~cm}$ de perímetro).

Los datos obtenidos fueron procesados con el paquete estadístico PRIMER 6 \& PERMANOVA + v.1.0.1. mediante un análisis univariante de la varianza por permutaciones (PERANOVA) (Anderson 2001). Para ello se utilizó un diseño de dos vías considerando los factores: localidad (fijo y con dos niveles: 1 . Punta del Hidalgo, 2. Finca El Apio) y hábitat (fijo y con dos niveles: 1. rocas pequeñas y 2. rocas grandes). Se usaron las distancias euclídeas para el cálculo de la matriz triangular. El análisis se llevó a cabo estableciendo un nivel de significación de $\alpha=0,05$. De manera adicional, se realizó un análisis de componentes principales (PCO) para estudiar la relación entre la abundancia de las especies y los distintos hábitats muestreados. 
También se realizó una regresión lineal curvilínea con el paquete estadístico SPSS (IBM) para estudiar la relación entre las variables talla y peso, con el fin de estimar la biomasa de los hábitats estudiados. Con la ecuación de la curva de regresión se calcularon los pesos del resto de cangrejos muestreados y se estimó la biomasa promedio (en $\mathrm{g} / \mathrm{m}^{2}$, con un esfuerzo de 30 minutos y cuatro personas) para cada uno de los hábitats.

\section{RESULTADOS}

\subsection{ESTADÍ́STICA DESCRIPTIVA GENERAL}

Se obtuvo un total de 339 individuos entre la Punta del Hidalgo $(\mathrm{n}=211)$ y la Finca El Apio ( $\mathrm{n}=128)$, de los cuales 17 individuos se corresponden con la especie Percnon gibbesi, 185 con Pachygrapsus spp., 90 con Xantho spp. y 47 con Porcellana platycheles (Pennant, 1777). En la tabla 1 y en la figura 3 se muestra el número de individuos muestreados para cada especie en función del sexo y de la localidad. Para el género Pachygrapsus se recolectó mayor número de machos tanto en la Punta del Hidalgo como en la Finca El Apio, observando la misma situación para las especies de Xantho. En contraposición, se encontró mayor número de hembras de $P$. platycheles en relación con los machos. De los 17 ejemplares de $P$. gibbesi, 16 fueron muestreados en la Punta del Hidalgo y un individuo en la Finca El Apio.

\begin{tabular}{|c|c|c|c|c|c|}
\hline \multicolumn{6}{|c|}{$\begin{array}{l}\text { TABLA 1. NÚMERO DE CANGREJOS MUESTREADOS Y ESTADÍSTICA DESCRIPTIVA } \\
\text { DE LA TALLA }(\mathrm{mm}) \text {, ATENDIENDO A LA LOCALIDAD Y AL SEXO DE LOS INDIVIDUOS }\end{array}$} \\
\hline & TotaL & Pachygrapsus spp. & Percnon gibbesi & Xantho spp. & Porcellana platycheles \\
\hline \multirow{5}{*}{$\begin{array}{c}\mathscr{\mathscr { U }} \\
\tilde{\tilde{J}} \\
\end{array}$} & Machos & 114 & 9 & 61 & 8 \\
\hline & Hembras & 68 & 8 & 27 & 36 \\
\hline & Indeterminados & 3 & 0 & 2 & 3 \\
\hline & Hembras ovígeras & 6 & 1 & 2 & 15 \\
\hline & Total & 185 & 17 & 90 & 47 \\
\hline \multirow{4}{*}{ 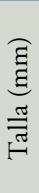 } & Media & 10,43 & 18,80 & 11,55 & 5,53 \\
\hline & Desviación estándar & 4,75 & 4,92 & 4,63 & 1,95 \\
\hline & Minimo & 2,40 & 10 & 2 & 2 \\
\hline & Máximo & 35,90 & 29,20 & 22 & 9,11 \\
\hline \multirow{6}{*}{ 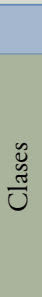 } & Punta del Hidalgo & Pachygrapsus spp. & Percnon gibbesi & Xantho spp. & Porcellana platycheles \\
\hline & Machos & 61 & 9 & 48 & 5 \\
\hline & Hembras & 40 & 7 & 21 & 14 \\
\hline & Indeterminados & 1 & 0 & 2 & 3 \\
\hline & Hembras ovígeras & 3 & 1 & 2 & 5 \\
\hline & Total & 102 & 16 & 71 & 22 \\
\hline
\end{tabular}




\begin{tabular}{|c|c|c|c|c|c|}
\hline \multirow{4}{*}{ 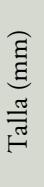 } & Media & 11,62 & 19,06 & 11,25 & 5,34 \\
\hline & Desviación estándar & 5,52 & 4,96 & 4,70 & 2,02 \\
\hline & Mínimo & 2,40 & 10 & 2 & 2 \\
\hline & Máximo & 35,90 & 29,20 & 22 & 9,11 \\
\hline \multirow{6}{*}{ 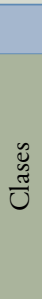 } & Finca El Apio & Pachygrapsus spp. & Percnon gibbesi & Xantho spp. & Porcellana platycheles \\
\hline & Machos & 53 & 0 & 13 & 3 \\
\hline & Hembras & 28 & 1 & 6 & 22 \\
\hline & Indeterminados & 2 & 0 & 0 & 0 \\
\hline & Hembras ovígeras & 3 & 0 & 0 & 10 \\
\hline & Total & 83 & 1 & 19 & 25 \\
\hline \multirow{4}{*}{ 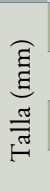 } & Media & 8,96 & 14,60 & 12,67 & 5,70 \\
\hline & Desviación estándar & 3,02 & - & 4,26 & 1,90 \\
\hline & Mínimo & 3 & 14,60 & 4 & 2 \\
\hline & Máximo & 18 & 14,60 & 21 & 9 \\
\hline
\end{tabular}

Con respecto a la talla, la especie Percnon gibbesi y el género Pachygrapsus alcanzan mayores valores de talla media en la Punta del Hidalgo en relación con la Finca El Apio, mientras que Xantho spp. y Porcellana platycheles mostraron tallas similares entre ambas localidades.

Se registró un total de seis hembras ovígeras para Pachygrapsus spp., una para Percnon gibbesi, dos para Xantho spp. y quince para Porcellana platycheles. Atendiendo a la tabla 2, la talla media es de 14,22 mm para Pachygrapsus spp., 13,85 mm para Xantho spp. y $5,53 \mathrm{~mm}$ para $P$. platycheles. La única hembra ovígera recolectada de la especie $P$. gibbesi presentó $29,2 \mathrm{~mm}$.

\begin{tabular}{|c|c|c|c|c|}
\hline \multicolumn{5}{|c|}{ TALLA DE HEMBRAS OVÍGERAS $(\mathrm{mm})$} \\
\hline EsPecies & Pachygrapsus spp. & Xantho spp. & Percnon gibbesi & Porcellana platycheles \\
\hline Media & 14,22 & 13,85 & 29,20 & 5,53 \\
\hline Desviación estándar & 5,46 & 0,21 & - & 1,46 \\
\hline Mínimo & 9 & 13,70 & 29,20 & 4 \\
\hline Máximo & 22 & 14 & 29,20 & 9 \\
\hline Total & 6 & 2 & 1 & 15 \\
\hline
\end{tabular}

\subsection{ANÁlisis univariante de LA VARIANZa POR PERMUtACIONES (PERMANOVA)}

Analizando todas las especies en conjunto, la diferencia de abundancia se mostró significativa para el hábitat ( $\mathrm{p}$-valor $=0,031)$, mientras que el factor localidad resultó no significativo ( $\mathrm{p}$-valor= 0,682$)$ (tabla 3 ). 

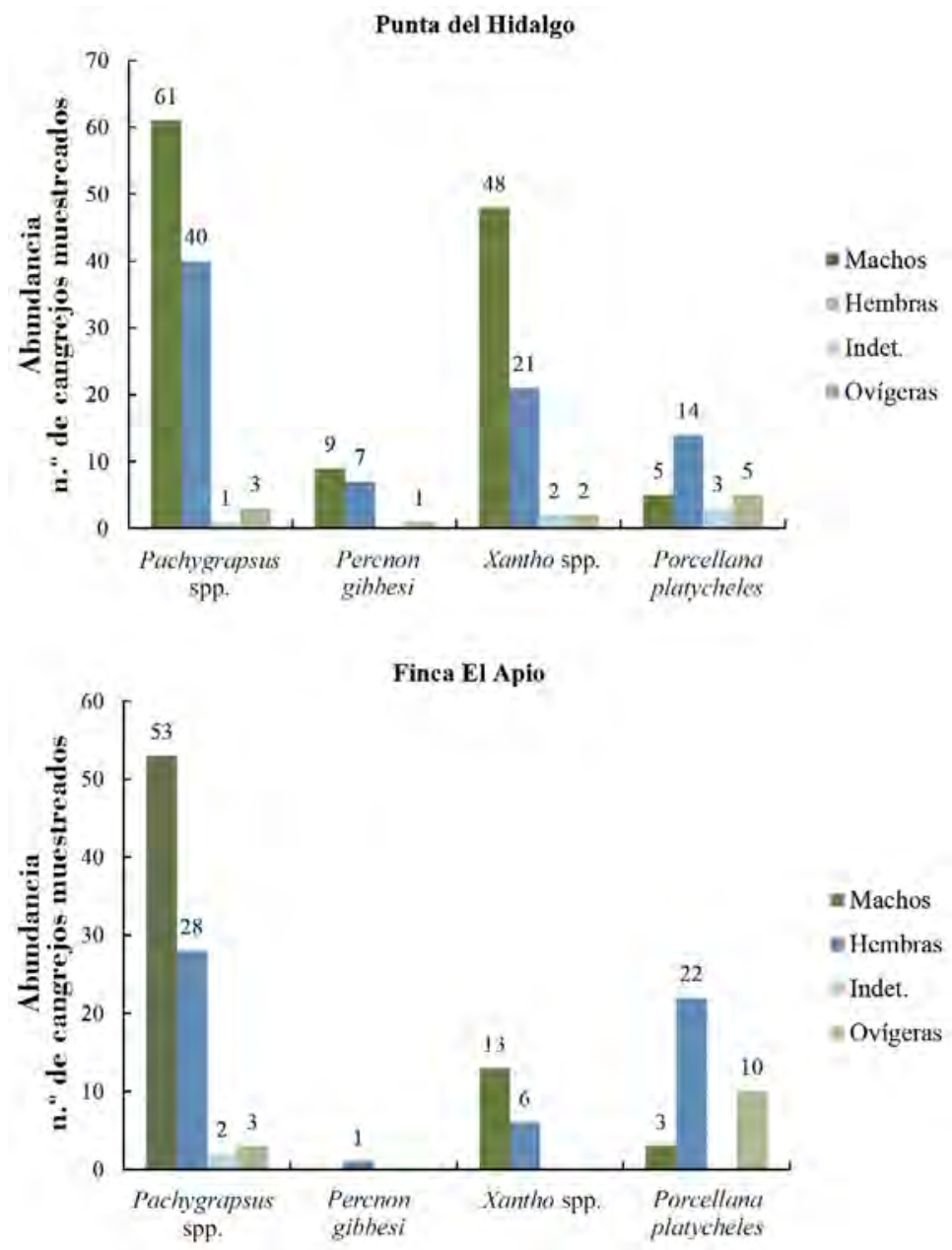

Figura 3. Número de cangrejos en función del sexo para ambas localidades.

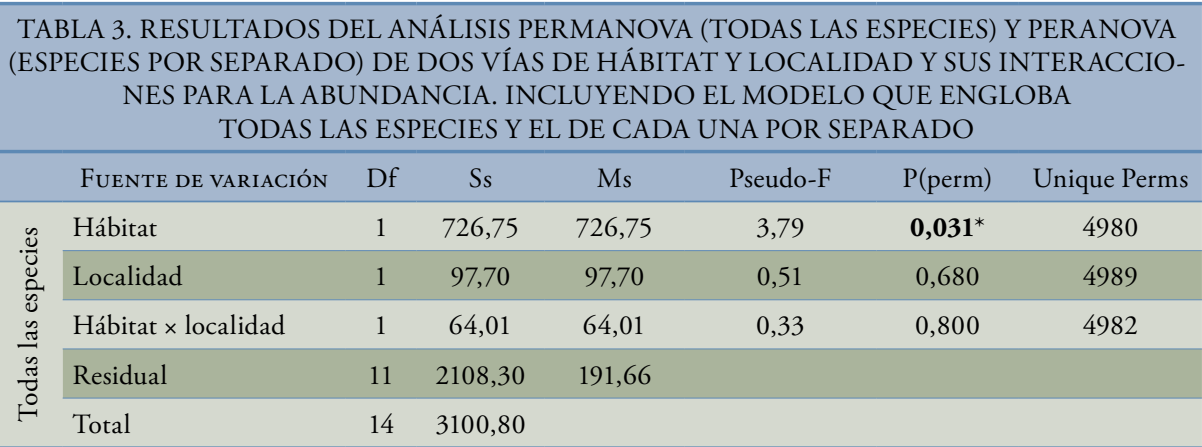




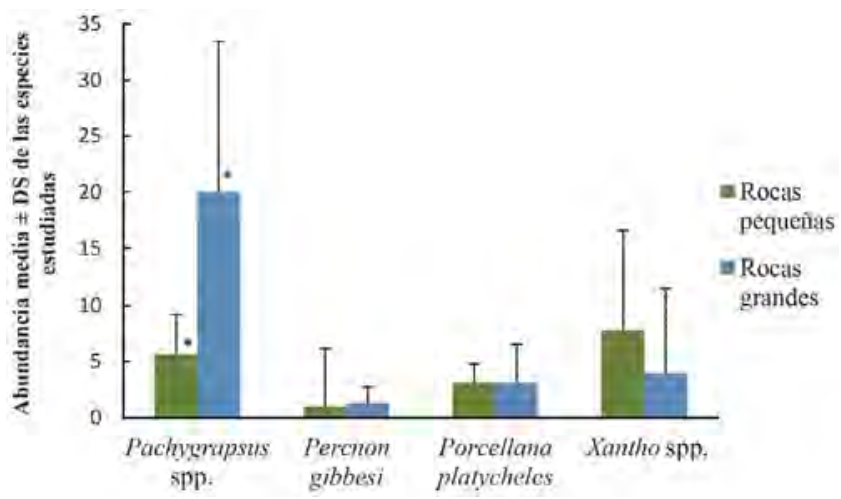

Figura 4. Abundancia media \pm DS de las especies estudiadas con respecto al tipo de hábitat. El asterisco $\left(^{*}\right)$ indica diferencias significativas.

\begin{tabular}{|c|c|c|c|c|c|c|c|}
\hline \multirow{5}{*}{$\begin{array}{l}\dot{0} \\
\text { के } \\
\text { sँ } \\
\text { है } \\
\text { है } \\
\text { है } \\
\text { है } \\
\text { है }\end{array}$} & Hábitat & 1 & 698,75 & 698,75 & 6,67 & $0,030^{*}$ & 4939 \\
\hline & Localidad & 1 & 9,56 & 9,56 & $9,12 \mathrm{E}-2$ & 0,760 & 4936 \\
\hline & Hábitat $\times$ localidad & 1 & 9,56 & 9,56 & $9,12 \mathrm{E}-2$ & 0,770 & 4958 \\
\hline & Residual & 11 & 1151,90 & 104,72 & & & \\
\hline & Total & 14 & 1943,30 & & & & \\
\hline \multirow{5}{*}{ 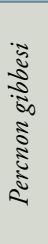 } & Hábitat & 1 & 0,24 & 0,24 & $3,34 \mathrm{E}-2$ & 0,880 & 2956 \\
\hline & Localidad & 1 & 9,85 & 9,85 & 1,38 & 0,290 & 1684 \\
\hline & Hábitat $\times$ localidad & 1 & 1,25 & 1,25 & 0,17 & 0,720 & 4659 \\
\hline & Residual & 11 & 78,62 & 7,15 & & & \\
\hline & Total & 14 & 89,73 & & & & \\
\hline \multirow{5}{*}{ 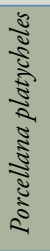 } & Hábitat & 1 & 0,18 & 0,18 & $7,36 \mathrm{E}-3$ & 0,930 & 4680 \\
\hline & Localidad & 1 & 10,25 & 10,25 & 0,42 & 0,520 & 4461 \\
\hline & Hábitat $\times$ localidad & 1 & 2,15 & 2,15 & $8,74 \mathrm{E}-2$ & 0,770 & 4920 \\
\hline & Residual & 11 & 270,88 & 24,63 & & & \\
\hline & Total & 14 & 283,73 & & & & \\
\hline \multirow{5}{*}{$\begin{array}{l}\dot{0} \\
\text { के } \\
\dot{\Sigma} \\
\frac{1}{2} \\
\text { है }\end{array}$} & Hábitat & 1 & 27,58 & 27,55 & 0,50 & 0,54 & 4925 \\
\hline & Localidad & 1 & 68,04 & 68,04 & 1,23 & 0,32 & 4921 \\
\hline & Hábitat $\times$ localidad & 1 & 51,05 & 51,05 & 0,92 & 0,39 & 4968 \\
\hline & Residual & 11 & 606,88 & 55,17 & & & \\
\hline & Total & 14 & 784 & & & & \\
\hline
\end{tabular}

* Factores significativos con valor de p(perm) en negrita.

Posteriormente se procedió a analizar las especies de forma independiente (PERANOVA), no existiendo diferencias significativas para la abundancia de Percnon gibbesi, Porcellana platycheles y Xantho spp. en cuanto a la localidad, ni al hábitat 
(tabla 3). En el caso de Pachygrapsus spp. se observaron diferencias significativas en el hábitat en cuanto a abundancia (figura 4), siendo mayor en aquellos lugares donde se cuente con mayor proporción de rocas grandes.

Para el estudio de la talla no se detectaron diferencias significativas para ninguna de las especies en relación con los factores establecidos (tabla 4).

\begin{tabular}{|c|c|c|c|c|c|c|c|}
\hline \multicolumn{8}{|c|}{$\begin{array}{l}\text { TABLA 4. RESULTADOS DEL ANÁLISIS PERANOVA DE DOS VÍAS DE HÁBITAT } \\
\text { Y LOCALIDAD Y SUS INTERACCIONES PARA LA TALLA. INCLUYENDO } \\
\text { EL MODELO PARA CADA ESPECIE POR SEPARADO }\end{array}$} \\
\hline \multirow{6}{*}{$\begin{array}{l}\dot{0} \\
\text { के } \\
\hat{z} \\
\text { है } \\
\text { हे } \\
\text { हे } \\
\text { हे } \\
\text { हे }\end{array}$} & FUENTE DE VARIACIÓN & Df & Ss & Ms & Pseudo-F & $\mathrm{P}($ perm $)$ & Unique Perms \\
\hline & Hábitat & 1 & 0,98 & 0,98 & $5,63 \mathrm{E}-2$ & 0,83 & 4957 \\
\hline & Localidad & 1 & 7,10 & 7,10 & 0,4067 & 0,55 & 4954 \\
\hline & Hábitat $\times$ localidad & 1 & $4,28 \mathrm{E}-2$ & $4,28 \mathrm{E}-2$ & $2,45 \mathrm{E}-3$ & 0,96 & 4950 \\
\hline & Residual & 11 & 192,10 & 17,46 & & & \\
\hline & Total & 14 & 200,04 & & & & \\
\hline \multirow{5}{*}{ 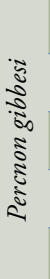 } & Hábitat & 1 & 81,50 & 81,50 & 1,11 & 0,32 & 3919 \\
\hline & Localidad & 1 & 84,96 & 84,96 & 1,16 & 0,30 & 3177 \\
\hline & Hábitat $\times$ localidad & 1 & $3,35 \mathrm{E}-2$ & $3,35 \mathrm{E}-2$ & $4,58 \mathrm{E}-4$ & 0,98 & 4856 \\
\hline & Residual & 11 & 803,41 & 73,04 & & & \\
\hline & Total & 14 & 982,20 & & & & \\
\hline \multirow{5}{*}{ 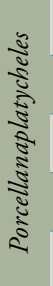 } & Hábitat & 1 & 3,08 & 3,08 & 0,32 & 0,58 & 4807 \\
\hline & Localidad & 1 & 0,38 & 0,38 & $3,99 \mathrm{E}-2$ & 0,84 & 4726 \\
\hline & Hábitat $\times$ localidad & 1 & 0,38 & 0,38 & $3,96 \mathrm{E}-2$ & 0,85 & 4928 \\
\hline & Residual & 11 & 104,80 & 9,53 & & & \\
\hline & Total & 14 & 108,26 & & & & \\
\hline \multirow{5}{*}{ 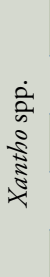 } & Hábitat & 1 & 2,51 & 2,51 & $6,46 \mathrm{E}-2$ & 0,80 & 4942 \\
\hline & Localidad & 1 & 81,54 & 81,54 & 2,09 & 0,17 & 4936 \\
\hline & Hábitat $\times$ localidad & 1 & 58,81 & 58,81 & 1,51 & 0,22 & 4954 \\
\hline & Residual & 11 & 428,36 & 38,94 & & & \\
\hline & Total & 14 & 569,87 & & & & \\
\hline
\end{tabular}

\subsection{AnÁlisis de COMponentes PRincipales (PCO)}

El análisis PCO mostró que en aquellas zonas donde hay una mayor abundancia de Pachygrapsus se registra una menor abundancia de Xantho. Los individuos relativos al género Xantho muestran preferencia por hábitats donde predominan 

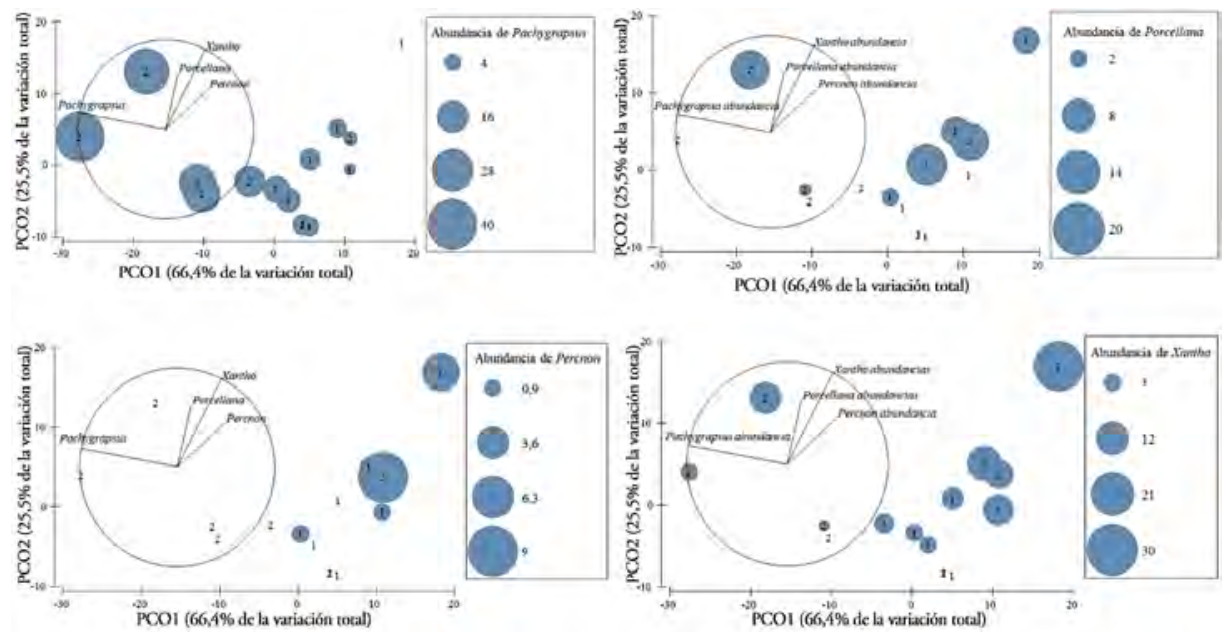

Figura 5. Ordenación PCO utilizando las abundancias de todas las especies muestreadas, mostrando la abundancia de cada una de las especies con burbujas y seńalando el hábitat con códigos ( 1 = rocas pequeñas; 2 = rocas grandes $)$.

rocas pequeñas. De la misma manera, se observa cómo Percnon gibbesi y Porcellana platycheles no presentan preferencias en cuanto al hábitat (figura 5).

\subsection{REgRESiÓN TALLA-PESO}

Las regresiones talla-peso para los géneros Xantho y Pachygrapsus muestran un buen ajuste a una curva logarítmica con un valor de significación de $\mathrm{p}<0,0001$, en ambos casos (figura 6).

\subsection{CÁlculo de biomasa}

En la tabla 5 se muestran los valores de biomasa estimados para cada tipo de hábitat, observándose mayores valores en el hábitat con mayor número de rocas pequeñas.

\begin{tabular}{|c|c|c|}
\hline \multicolumn{3}{|c|}{$\begin{array}{l}\text { TABLA 5. BIOMASA ESTIMADA }\left(\mathrm{g} / \mathrm{m}^{2}\right) \text { PARA LOS GÉNEROS XANTHO } \\
\text { Y PACHYGRAPSUS PARA LOS DISTINTOS HÁBITATS ESTUDIADOS }\end{array}$} \\
\hline Biomasa $\left(\mathrm{g} / \mathrm{m}^{2}\right)$ & ROCAS PEQUEÑAS & Rocas GRandes \\
\hline Xantho spp. & 0,10 & 0,08 \\
\hline Pachygrapsus spp. & 0,09 & 0,09 \\
\hline
\end{tabular}




\section{Pachygrapsus spp.}

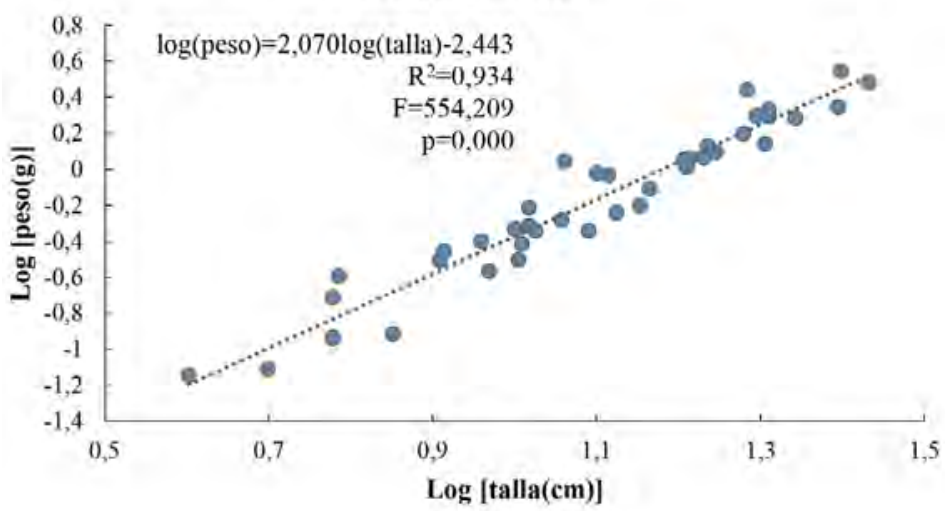

Xantho spp.

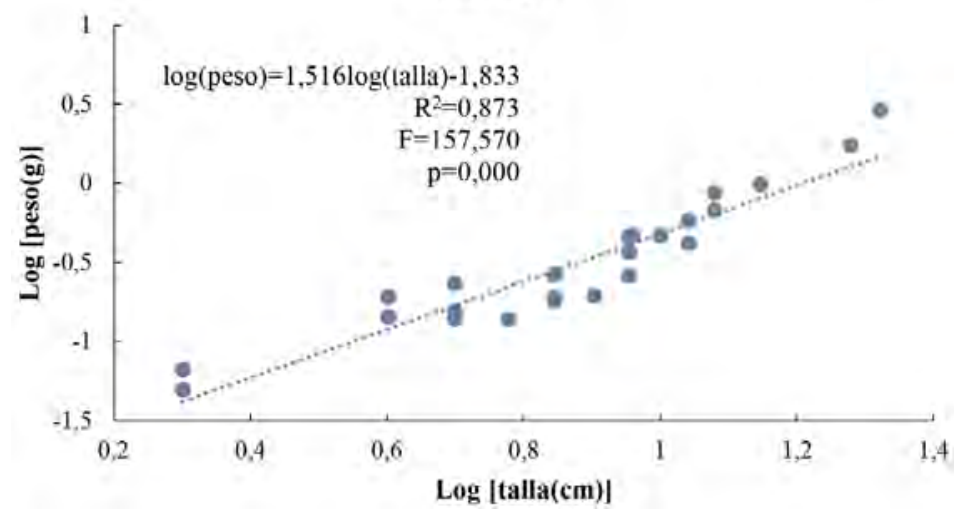

Figura 6. Gráficos de dispersión para la talla y el peso de los ejemplares de los géneros Pachygrapsus y Xantho. La recta representa la línea de ajuste de la regresión logarítmica. Se incluye la ecuación de la recta, el valor de $\mathrm{R}^{2}$, F y el p-valor.

\section{DISCUSIÓN}

La sobreexplotación de los recursos marisqueros es una de las principales alteraciones del litoral de las islas Canarias (Bonnet y Rodríguez 1992; González 1995; Noguera y Riera 2011). Debido a la escasez de datos previos, el presente estudio pretende determinar si las poblaciones de los géneros Xantho, Pachygrapsus y Percnon utilizados como carnada están afectadas por el marisqueo mediante la comparación de dos localidades, asumiendo que en una de ellas se desarrolla mayor presión marisquera, debido a la facilidad de acceso que presenta con respecto a la otra.

De los individuos muestreados, se encontró un mayor número de machos en ambas localidades para el género Pachygrapsus, lo que concuerda con los resultados 
de Flores y Negreiros-Franzoso (1999) y Flores y Paula (2002a), que muestran una proporción de sexos desviada hacia los machos para las clases de tallas mayores de 10 $\mathrm{mm}$. Para Xantho también se encontró un mayor número de machos, un resultado distinto al de Spivak et al. (2010), que encontraron proporciones de sexo muy próximas a 1:1. En Porcellana platycheles se da el caso contrario, con un mayor número de hembras, mientras que para Percnon gibbesi la proporción de machos es similar a la de hembras. La proporción de hembras ovígeras de Pachygrapsus y Xantho parece preocupantemente baja si se tienen en cuenta el número total de hembras y que el muestreo se realizó durante el periodo de veda, establecido para no interferir con el periodo de desove (BOC n. ${ }^{\circ}$ 093, 2 de mayo 2011). Sin embargo, este resultado es consistente con diversos estudios que indican que el periodo reproductivo de estas especies se produce en los meses de primavera y verano (Flores y Negreiros-Franzoso 1999; Flores y Paula 2002a: Paula y Flores 2002b; Spivak et al. 2010; Noguera y Riera 2011).

En cuanto a la talla, Percnon gibbesi y el género Pachygrapsus registran mayores valores de talla media en la Punta del Hidalgo mientras que Xantho spp. y Porcellana platycheles muestran valores similares de talla media en las dos localidades, aunque los análisis estadísticos muestran que no existen diferencias significativas para la talla entre las distintas localidades.

En el análisis que contempla todas las especies en conjunto no se encontraron diferencias significativas para la abundancia en cuanto a las localidades, pero sí en cuanto al hábitat; por tanto, la abundancia de las poblaciones no se ve afectada a priori por el marisqueo, aunque este resultado no es del todo concluyente ya que el muestreo se realizó durante los meses de veda. En los modelos de cada especie, Pachygrapsus presenta diferencias de abundancia en función del hábitat, observando una mayor presencia en aquellos hábitats de rocas de tamaño grande. En el caso de Xantho, Percnon y Porcellana, no existen diferencias significativas de abundancia para el hábitat.

Las diferencias a nivel de hábitat se observan en los gráficos del análisis PCO para la abundancia de las distintas especies en función del hábitat, observándose cómo a mayor abundancia de Pachygrapsus, menor abundancia de Xantho. Esto puede ser debido a una competencia por el hábitat. No obstante, para una interpretación más ajustada de estos resultados, sería recomendable un estudio experimental más detallado de las dos especies. Por el contrario, Porcellana platichelles y Percnon gibbesi no siguen una tendencia definida por el hábitat. A partir de los resultados obtenidos por el PCO, se muestra una mayor preferencia de Xantho por rocas pequeñas. Estos resultados se contradicen con los estudios de Spivak et al. (2010), que determinaron una preferencia por rocas grandes (más de $513 \mathrm{~cm}^{2}$ de superficie) sobre guijarros. Esto podría deberse a la diferencia en la toma de datos, así como a la elección de clasificación del hábitat.

Con los datos de biomasa $\left(\mathrm{g} / \mathrm{m}^{2}\right)$ existen mayores diferencias para Xantho entre hábitats, que para Pachygrapsus. Además, se destaca que en el hábitat dominado por rocas pequeñas se alcanzan mayores valores de biomasa para ambas especies. Atendiendo a la legislación vigente, el volumen máximo de extracción permitido para estas especies de cangrejo (1500 g/día de Xantho y 500 g/día de Pachygrapsus y 
Percnon en el caso de marisqueo profesional; $200 \mathrm{~g} /$ día de cada una de las especies para marisqueo recreativo) resulta excesivo atendiendo a la biomasa estimada para este recurso pesquero. Para la recolección de las cantidades máximas, el área de marisqueo se extendería varios kilómetros a lo largo del litoral. Además, teniendo en cuenta la presión del marisqueo y que el cese de la veda se produce en el inicio del periodo reproductivo, las poblaciones de estos cangrejos podrían experimentar una fuerte regresión. Como indica González (2016), en algunas localidades las poblaciones de estas especies se encuentran en estado crítico, por lo que futuros estudios que se prolonguen en el tiempo y que abarquen periodos dentro y fuera de la veda podrían demostrar claramente que las poblaciones se encuentran realmente en peligro, y de esta forma promover la implementación de medidas de conservación eficaces.

ReCiBIDO: octubre de 2017, ACEPTADO: noviembre de 2017

\section{AGRADECIMIENTOS}

Agradecemos a la comisión académica del Máster de Biología Marina: Biodiversidad y Conservación de la Universidad de La Laguna la oportunidad de realizar este estudio piloto para la asignatura de «Impacto de la Pesca en la Biodiversidad Marina». Agradecemos a Daniel Miranda González su colaboración durante los muestreos y a José Carlos Hernández toda la ayuda prestada.

\section{CONTRIBUCIÓN DE LOS AUTORES}

Conceptualización: AF.

Metodología: AF, MBS, AMH, DAC, NC.

Análisis de datos: DAC, AMH con la ayuda del profesor José Carlos Hernández. Investigación: AF, MBS, AMH, DAC, NC.

Preparación del escrito original: AF, MBS, AMH, DAC, NC.

Corrección y edición del escrito definitivo: AF, MBS, AMH, DA. 


\section{REFERENCIAS}

Alfonso, B., Sarabia, A., Sancibrián, I., Alfaro, A., Adern, N. y Hernández, J.C. 2015. Efecto de la actividad humana sobre la distribución y estructura poblacional del burgado Phorcus sauciatus (Koch, 1845). Rev. Acad. Canar. Cienc. 27: 333-343.

Anderson, M.J. 2001. A new method for non-parametric multivariate analysis of variance. Austral Ecol. 26(1): 32-46.

Bonnet, J. y Rodríguez, A. 1992. Fauna marina amenazada en las islas Canarias. Madrid: Instituto para la Conservación del Medio Ambiente (ICONA).

Deudero, S., Frau, A., Cerda, M. y Hampel, H. 2005. Distribution and densities of the decapod crab Percnon gibbesi, an invasive Grapsidae, in western Mediterranean waters. Mar. Ecol. Prog. Ser. 285: 151-156.

FEnberg, PB. y Roy, K. 2008. Ecological and evolutionary consequences of size-selective harvesting: how much do we know? Mol. Ecol. 17(1): 209-220.

Ferns, P.N., Rostron, D.M. y Siman, H.Y. 2000. Effects of mechanical cockle harvesting on intertidal communities. J. Appl. Ecol. 37(3): 464-474.

Flores, A.A. y Paula, J. 2002a. Population dynamics of the shore crab Pachygrapsus marmoratus (Brachyura: Grapsidae) in the central Portuguese coast. J. Mar. Biol. Assoc. U.K. 82(02): 229-241.

Flores, A.A. y Paula, J. 2002b. Sexual maturity, larval release and reproductive output of two brachyuran crabs from a rocky intertidal area in central Portugal. Invert. Reprod. Dev. 42(1): 21-34.

Flores, A.A. y Negreiros-Fransozo, M.L. 1999. On the population biology of the mottled shore crab Pachygrapsus transversus (Gibbes, 1850) (Brachyura, Grapsidae) in a subtropical area. Bull. Mar. Sci. 65(1): 59-73.

Freire, J. y García-Allut, A. 2000. Socioeconomic and biological causes of management failures in European artisanal fisheries: the case of Galicia (NW Spain). Mar. Policy. 4(5): 375-384.

González, J.A. 1995. Catálogo de los crustáceos decápodos de las Islas Canarias. Tenerife: Publicaciones Turquesa $273 \mathrm{p}$.

GonzÁLEz, J.A. 2016. Brachyuran crabs (Crustacea: Decapoda) from the Canary Islands (eastern Atlantic): checklist, zoogeographic considerations and conservation. Sci. Mar. 80(1): 89-102.

Noguera, R. y Riera, R. 2011. Dinámica de las poblaciones de Xantho spp. (cangrejilla) (Decapoda, Xanthidae) en la franja costera de Arrecife (Lanzarote, islas Canarias). Vieraea. (39): 97-104.

Paula, J. 1989. Rhythms of larval release of decapod crustaceans in the Mira Estuary, Portugal. Mar. Biol. 100(3): 309-312.

Sciberras, M. y Schembri, P.J. 2007. Observations on the alien crab Percnon gibbesi (Decapoda, Brachyura, Grapsidae) from the Maltese Islands. Rapp. P.-V. Reun. Comm. Int. Explor. Sci. Mer. Mediterr. 38: 594.

Spivak, E.D., Arévalo, E., Cuesta, J.A. y González-Gordillo, J.I. 2010. Population structure and reproductive biology of the stone crab Xantho poressa (Crustacea: Decapoda: Xanthidae) in the «Corrales de Rota» (south-western Spain), a human-modified intertidal fishing area. J. Mar. Biol. Assoc. U.K. 90(2): 323-334. 